Pacific Journal of Mathematics

CHARACTERIZATION OF HOMOGENEOUS SPACES AND 


\title{
CHARACTERIZATION OF HOMOGENEOUS SPACES AND THEIR NORMS
}

\author{
J. A. WARD
}

Let $G$ denote a compact group and $B$ a homogeneous Banach space of pseudomeasures over $G$ ( $B$ is left translation invariant with continuous shifts). If $T(G)$ is the linear space of trigonometric polynomials defined on $G$ then $T(G) \cap B$ is a dense subspace of $B$. An explicit description is given of $T(G) \cap B$. A complete list of the homogeneous closed subspaces of $B$ is given. Moreover it is shown that $B$ is determined by the set $T(G) \cap B$ and $N$, the restriction of the $B$ norm to this set. This leads to a complete description of those subsets of $T(G)$ and norms $N$ which determine homogeneous Banach spaces.

Introduction. Homogeneous Banach spaces first appeared in the paper of G. Silov, [9], where they were introduced as classes of Banach algebras of functions (under pointwise multiplication) on compact abelian groups. Each has the underlying group as its maximal ideal space. In [6], $\mathrm{K}$. de Leeuw omitted this property before classifying all possible maximal ideal spaces of homogeneous Banach algebras, and determining a correspondence between these algebras and certain norms.

If pointwise multiplication becomes convolution (and the underlying group is required to be locally compact and abelian) then we have the Segal algebras introduced in [7] by $\mathrm{H}$. Reiter. The generalization of Segal algebras to homogeneous Banach algebras given by $\mathrm{H}$. C. Wang in [10] corresponds to de Leeuw's extension of Silov's algebras. J. T. Burnham generalized, in [1], Segal algebras to allow the underlying group to be non-abelian. See Burnham's discussion in [2] of the development of this subject.

In this paper we study homogeneous Banach spaces in which the underlying group is taken to be compact and non-abelian. The translation operator is taken to be the left translation operator. In $\$ 2$ we establish some fundamental properties of these spaces; each is an $M(G)$-Banach module, 2.2, and further each has a set of trigonometric polynomials as a dense subspace, 2.4. In 2.6 we give an explicit description of this subspace.

We use these results in $\$ 3$ to classify all the homogeneous subspaces of a homogeneous Banach space, 3.2. We also show in 3.3 that if every element of the space is a measure, then each element is in fact generated by an $L^{1}$-function. This allows us to conclude in 3.4 that every such space is actually a convolution algebra. 
In $\$ 4$ we obtain a characterization of those norms which determine homogeneous Banach spaces. In fact we establish a correspondence between homogeneous Banach spaces and norms on certain spaces of trigonometric polynomials (those found in 2.6). In [6] de Leeuw presents a similar result.

The results of $\$ 2$ can be used in another way. Combined with those announced by the author in [12] they give a complete picture of the closed ideal theory of any homogeneous Banach algebra (a homogeneous Banach space which is an algebra under convolution). In particular, it can be shown that its closed ideal structure is precisely the same as that of $L^{1}$ or one of its homogeneous subspaces. We present these results in [13].

Most of the material contained in this paper, and in [12] and [13], is found in [11].

1. Preliminaries. Let $G$ denote a compact group with dual object $\Sigma$, the set of equivalence classes of continuous irreducible unitary representations of $G$. For each $\sigma \in \Sigma$ we fix a representative $U_{\sigma}$ and denote by $H_{\sigma}$ the Hilbert space on which $U_{\sigma}$ acts. The finite dimension of $H_{\sigma}$ is $d_{\sigma}$. We write $E(\Sigma)=\prod_{\sigma \in \Sigma} B\left(H_{\sigma}\right)$ where $B\left(H_{\sigma}\right)$ is the space of bounded operators on $H_{\sigma}$. For each $A=\left(A_{\sigma}\right) \in E(\Sigma)$ and each $p \geq 1$ put

$$
\|A\|_{p}=\left(\sum_{\sigma \in \Sigma} d_{\sigma}\left\|A_{\sigma}\right\|_{\phi_{p}}^{p}\right)^{1 / p}
$$

and put

$$
\|A\|_{\infty}=\sup _{\sigma \in \Sigma}\left\|A_{\sigma}\right\|_{\phi_{\infty}}
$$

where \|\|$_{\phi_{p}}$ and \|\|$_{\phi_{\infty}}$ are the Von Neumann norms (see D.36 and D.37 of [4]). Then for $1 \leq p \leq \infty$,

$$
E_{p}(\Sigma)=\left\{A \in E(\Sigma):\|A\|_{p}<\infty\right\} \text {. }
$$

We also put

$$
E_{0}(\Sigma)=\left\{A \in E_{\infty}(\Sigma):\left\{\sigma \in \Sigma:\left\|A_{\sigma}\right\|_{\phi_{\infty}} \geq \varepsilon\right\} \text { is finite for all } \varepsilon>0\right\}
$$

and

$$
E_{00}(\Sigma)=\left\{A \in E(\Sigma):\left\{\sigma \in \Sigma: A_{\sigma} \neq 0\right\} \text { is finite }\right\} .
$$

The Fourier series of $\mu \in M(G)$ is given by

$$
\sum_{\sigma \in \Sigma} d_{\sigma} \operatorname{Tr}\left(\hat{\mu}(\sigma) U_{\sigma}(x)^{*}\right)
$$


where $\operatorname{Tr}$ is the usual trace function, and the Fourier-Stieltjes transform $\hat{\mu}(\sigma)$ of $\mu$ at $\sigma$ is given by

$$
\hat{\mu}(\sigma)=\int U_{\sigma}(x) d \mu(x) .
$$

Note that the Fourier transform $f \rightarrow \hat{f}$ provides an isometric isomorphism between the spaces $A(G)$ (of functions with absolutely convergent Fourier series) and $E_{1}(\Sigma)$.

We define $P(G)$, the space of pseudomeasures, to be the topological dual of $A(G)$. Clearly $M(G) \subseteq P(G)$. The 'Fourier transform' of an element $S$ of $P(G), \hat{S}$, is defined by requiring that $\hat{S}(\hat{f})=S(f)$ for all $f \in A(G)$. This definition is consistent with the definition of the Fourier Stieltjes transform if $S$ is an element of $M(G)$. Moreover the map $S \rightarrow \hat{S}$ is an isometric isomorphism between $P(G)$ and $E_{\infty}(\Sigma)$. The convolution product of two pseudomeasures $S$ and $T$ is the element $S * T$ of $P(G)$ defined by

$$
(S * T)^{\hat{n}}=\hat{S} \cdot \hat{T}
$$

where the product on the right side of (1) is the pointwise product of the elements $\hat{S}$ and $\hat{T}$ of $E_{\infty}(\Sigma)$. It is easily seen that $P(G)$ is a two-sided convolution module over $A(G)$. Finally, for each $x \in G$ and $S \in P(G)$ we define the left $x$-translate of $S$ to be the element ${ }_{x} S$ of $P(G)$ defined by

$$
{ }_{x} S(f)=S\left({ }_{x^{-1}} f\right) \text { for all } f \in A(G) .
$$

The definitions of convolution and left translates agree with the usual definitions when $S$ and $T$ are elements of $M(G)$.

In the first instance we consider subspaces of $P(G)$ which become Banach spaces when equipped with a suitable norm, and which are closed under left translation, each translation operator being continuous. Let $\left(B,\|\|_{B}\right.$ ) denote such a space. Then $B$ is homogeneous (or is a homogeneous Banach space) if the maps $x \rightarrow_{x} b$ of $G$ to $B$ are continuous at $e$, the identity of $G$, for each $b \in B$. Note that this ensures continuity at each $x \in G$. The more familiar examples of homogeneous Banach spaces are $A(G), C(G)$ and $L^{p}(G), 1 \leq p<\infty$; however neither $L^{\infty}(G)$ nor $M(G)$ are homogeneous. Nor is $P(G)$ itself. However its closed subalgebra $P F(G)$, of pseudomeasures whose Fourier transform vanish at infinity (sometimes called pseudofunctions), is homogeneous. Many more examples are presented in $\$ 5$.

2. Homogeneous Banach spaces. In this section we present some elementary results concerning homogeneous Banach spaces. The analogues, for $G$ an abelian group, are found in chapter 2 of [10]. Throughout 
this section $B$, equipped with norm \|\|$_{B}$, denotes a homogeneous Banach space unless otherwise stated. We show that convolution on the right by an element of $M(G)$ produces a continuous endomorphism of $B$ (2.2); moreover, any bounded approximate identity $\left(f_{n}\right)_{n \in \mathbf{N}}$ of $L^{1}(G)$, with $\left\|f_{n}\right\|_{L^{1}}=1$ for all $n$, acts like an approximate identity of $B$, (2.3). This leads us to the characterization (2.4) of homogeneous spaces, and further to the density of $T(G) \cap B$ in $B$.

\subsection{LemMA. There exists a positive constant $K$ such that}

$$
\sup _{x \in G}\left\|_{x} b\right\|_{B} \leq K\|b\|_{B} \quad \text { for all } b \in B .
$$

Proof. This is an immediate consequence of the continuity of the shifts $x \rightarrow{ }_{x} b$ from $G$ to $B$ and of the compactness of $G$.

2.2 Proposition. $B$ is an $M(G)$-Banach module. That is, for each $\mu \in M(G)$ and $b \in B, \mu * b$ is an element of $B$, and

$$
\|\mu * b\|_{B} \leq K\|\mu\|_{M}\|b\|_{B}
$$

where $K$ is determined as in 2.1.

Proof. The theory of integrals of vector-valued functions, as presented in [3], provides an easy proof because $\mu * b$ may be identified as the unique element of the algebraic dual of $B^{*}$ (the continuous dual of $B$ ) which satisfies

$$
\left\langle\mu * b, b^{*}\right\rangle=\int\left\langle b^{*},{ }_{x} b\right\rangle d \mu(x)
$$

for all $b^{*} \in B^{*}$. By 8.14.14 and 8.15.2 of [3] $\mu * b$ is in $B$, regarded as a subspace of the algebraic dual of $B^{*}$, if the following hold:

1. the map $g: G \rightarrow B, x \rightarrow{ }_{x} b$, is measurable and

2. $\int\|g(x)\|_{B} d \mu(x)$ is finite.

Now 1. is an immediate consequence of the homogeneity of $B$, and 2 . follows from 2.1. The inequality follows from 8.14.6 of [3].

Hewitt and Ross, in (32.23) and (32.48) of [4], discuss a number of cases in which an approximate identity in a Banach algebra $A$ acts like an approximate identity in a Banach $A$-module. In 2.3 we present a further case in which $A$ is taken to be $L^{1}(G)$ and we consider $B$ a homogeneous Banach space. It is a consequence of 2.2 that $B$ is a Banach $L^{1}(G)$-module. 
We let $\left(f_{n}\right)_{n \in \mathrm{N}}$ denote a bounded left approximate identity of $L^{1}(G)$ for which $\left\|f_{n}\right\|_{L^{1}}=1$ for all $n$. By (28.52) of [4] we have $\lim _{n \rightarrow \infty}\left\|f_{n} \chi_{G \backslash U}\right\|_{L^{1}}=0$ for every neighborhood $U$ of the identity of $G\left(\chi_{V}\right.$ denotes the characteristic function of the subset $V$ of $G$; that is, $\chi_{V}(x)=1$ if $x \in V$ and $\chi_{V}(x)=0$ if $\left.x \in G \backslash V\right)$.

2.3 TheOREM. $\lim _{n \rightarrow \infty}\left\|f_{n} * b-b\right\|_{B}=0$ for all $b \in B$.

Proof. By $2.2 f_{n} * b$ is an element of $B$ for each $n$. We write

$$
f_{n} * b-b=\int f_{n}(x)\left({ }_{x} b-b\right) d \lambda(x)
$$

where $\lambda$ denotes the normalized left invariant Haar measure on $G$. Fix $\varepsilon>0$. There exists a neighborhood $U$ of the identity of $G$ such that for each $x \in U,\left\|_{x} b-b\right\|_{B}<\varepsilon$, and a positive integer $N$ such that for $n>N$,

$$
\left\|f_{n} \chi_{G \backslash U}\right\|_{L^{1}}<\varepsilon\left(\sup _{x \in G}\left\|_{x} b-b\right\|_{B}\right)^{-1} \text {. }
$$

Therefore, by (1) and 8.14 .6 of [3], for $n>N$

$$
\left\|f_{n} * b-b\right\|_{B}<\varepsilon\left(\left\|f_{n}\right\|_{L^{1}}+1\right)=2 \varepsilon
$$

and so $\lim _{n \rightarrow \infty}\left\|f_{n} * b-b\right\|_{B}=0$.

It is a consequence of 2.3 that $L^{1}(G) * B$ is a dense subspace of $B$. Furthermore, since the $f_{n}$ 's may be taken from $T(G)$ (see (28.57) of [4]), we have that $B \cap T(G)$ is a dense subspace of $B$.

2.4 Corollary. $B \cap T(G)$ is a dense subspace of $B$.

We now provide a partial converse to 2.3.

In 2.5 let $B$ denote a translation invariant Banach space of pseudomeasures in which each translation operator is continuous. If the map $x \rightarrow{ }_{x} b$ is a measurable function of $G$ into $B$ for each $b \in B$, then the argument used in 2.2 also shows that $B$ is a Banach $M(G)$-module.

2.5 THEOREM. If there exists a left approximate identity $\left(f_{n}\right)_{n \in \mathbf{N}}$ of $L^{1}(G)$, taken from $T(G)$, such that

$$
\lim _{n \rightarrow \infty}\left\|f_{n} * b-b\right\|_{B}=0 \quad \text { for all } b \in B
$$

then $B$ is homogeneous. 
Proof. For each $n \in \mathbf{N}, b \in B$ and $x \in G$ we have

$$
\left\|f_{n} * b-f_{n} * b\right\|_{B} \leq K\left\|_{x} f_{n}-f_{n}\right\|_{L^{\prime}}\|b\|_{B}
$$

and so the map $x \rightarrow{ }_{x} f_{n} * b$ is continuous at the identity $e$ of $G$.

$B$ is homogeneous if for each $b \in B$, the map $T_{b}: x \rightarrow_{x} b$ is continuous at $e$. In view of (1) we have that $T_{b}$ is continuous at $e$ for each $b$ in the dense subset $\left\{f_{n} * b^{\prime}: n \in \mathbf{N}, b^{\prime} \in B\right\}$ of $B$.

Let $b \in B$. The set of discontinuities of $T_{b}$ must be of the first category by the Baire Category Theorem, and so is not the whole of $G$. The compactness of $G$ ensures that $T_{b}$ is continuous at $e$. To see this, let $x$ denote a point of continuity of $T_{b}$. If $e$ is known to be such a point then the proof is complete; if we are uncertain fix $\varepsilon>0$. There exists a neighborhood $U$ of $x$ so that for $y \in U,\left\|_{y} b-{ }_{x} b\right\|_{B}<\varepsilon$. Now let $V$ denote the set of left $x^{-1}$-translates of the elements of $U$. Then $V$ is a neighborhood of $e$. Moreover, for each $y \in V$

$$
\begin{aligned}
\|y-b\|_{B} & =\left\|x_{x^{-1} x y} b-{ }_{x^{-1} x} b\right\|_{B} \\
& \leq \text { const }\left\|_{x y} b-{ }_{x} b\right\|_{B}<\varepsilon \cdot \text { const. }
\end{aligned}
$$

since $x y \in U$. Hence $e$ is a point of continuity of $T_{b}$.

We are able to give a specific description of the dense subspace $B \cap T(G)$ of $B$ when $B$ is homogeneous. First, however, we introduce some notation.

For each $\sigma \in \Sigma$ let $E_{\sigma}$ denote a subspace of $H_{\sigma}$. Denote by $\mathcal{E}$ the set $\mathcal{E}=\left\{E_{\sigma}: \sigma \in \Sigma\right\}$. For any subset $A$ of $P(G), A_{\mathscr{G}}$ denotes the set

$$
A_{\mathscr{\sigma}}=\left\{S \in A: E_{\sigma} \subseteq \operatorname{ker} \hat{S}(\sigma) \text { for each } \sigma \in \Sigma\right\} \text {. }
$$

In particular, if $E_{\sigma}=H_{\sigma}$ for each $\sigma \in \Sigma \backslash F(F \subseteq \Sigma)$ and if $E_{\sigma}=\{0\}$ for $\sigma \in F$ then $A_{\mathscr{\delta}}$ is the set of pseudomeasures in $A$ whose Fourier transform is supported by $F$; that is, $A_{\mathscr{E}}=A_{F}$.

2.6. Proposition. Assume $B$ is homogeneous. For each $\sigma \in \Sigma$ put $E_{\sigma}=\bigcap\{\operatorname{ker} \hat{b}(\sigma): b \in B\}$, and put $\mathcal{E}=\left\{E_{\sigma}: \sigma \in \Sigma\right\}$. Then $B \cap T(G)=$ $T(G)_{\mathscr{E}}$.

Proof. Obviously $B \cap T(G)$ is a subset of $T(G)_{\mathscr{E}}$.

To prove the reverse inclusion it is sufficient to prove that any $k \in T(G)_{\{\sigma\}}(\sigma \in \Sigma)$, satisfying $E_{\sigma} \subseteq \operatorname{ker} \hat{k}(\sigma)$, is in $B$.

We first prove that the set $\hat{B}(\sigma)$, which we define to be $\{\hat{b}(\sigma)$ : $b \in B\}$, is precisely $\left\{T \in B\left(H_{\sigma}\right): E_{\sigma} \subseteq \operatorname{ker} T\right\}$. The set $\hat{B}(\sigma)$ is, in fact, a 
left ideal of $B\left(H_{\sigma}\right)$. To see this we observe that by (38.32) of [4] there exist elements $x_{i} \in G, i=1, \ldots, d_{\sigma}^{2}$, such that for any $T \in B\left(H_{\sigma}\right)$ there are constants $\alpha_{i}(T), i=1, \ldots, d_{\sigma}^{2}$, satisfying $T=\sum_{i=1}^{d_{\sigma}^{2}} \alpha_{i}(T) U_{\sigma}\left(x_{i}\right)$. So, for each $b \in B$,

$$
T \hat{b}(\sigma)=\left(\sum_{i=1}^{d_{\sigma}^{2}} \alpha_{i}(T) U_{\sigma}\left(x_{i}\right)\right) \hat{b}(\sigma)=\sum_{i=1}^{d_{\sigma}^{2}} \alpha_{l}(T)_{x_{i}} \hat{b}(\sigma) .
$$

But $\sum_{i=1}^{d_{\sigma}^{2}} \alpha_{i}(T){ }_{x_{i}} b$ is in $B$, and so it follows that $\hat{B}(\sigma)$ is a left ideal of $B\left(H_{\sigma}\right)$. Then, by (38.11) of [4] $\hat{B}(\sigma)$ must equal $\left\{T \in B\left(H_{\sigma}\right): Y_{\sigma} \subseteq \operatorname{ker} T\right\}$ for some subspace $Y_{\sigma}$ of $H_{\sigma}$. Clearly $Y_{\sigma}$ must equal $E_{\sigma}$ as we required.

It follows that the operator $\hat{k}(\sigma)$ is in $\hat{B}(\sigma)$, and so there exists $b \in B$ such that $\hat{b}(\sigma)=\hat{k}(\sigma)$. Let $\chi_{\sigma}$ denote the trigonometric polynomial defined by $\chi_{\sigma}(x)=d_{\sigma} \operatorname{Tr}\left(U_{\sigma}(x)^{*}\right)$ for each $x \in G$. By $2.2 \chi_{\sigma} * b \in B$. But $\chi_{\sigma} * b$ must equal $k$ and so $k \in B$.

In (38.30) of [4] the authors give an explicit form for those linear subspaces of $T(G)$ which are closed under left translation. Since $B \cap T(G)$ is such a subspace we could have applied (38.30) to obtain 2.6.

3. Closed translation-invariant subspaces. Throughout the remainder of this paper we will assume that each homogeneous Banach space $B$ may be continuously embedded in $P(G)$; that is, there exists a constant $C$, depending only on $B$, such that $\|b\|_{P} \leq C\|b\|_{B}$ for all $b \in B$. (In fact, we require only that for each $\sigma \in \Sigma$ there exists a constant $C_{\sigma}$ such that $\|\hat{b}(\sigma)\|_{B\left(H_{\sigma}\right)} \leq C_{\sigma}\|b\|_{B}$ for all $b \in B$. However, a simple application of the Closed Graph Theorem demonstrates that these two conditions are equivalent.) All of the familiar homogeneous Banach spaces have this property.

We first consider the question of which Banach subspaces of $B$ are themselves homogeneous. Clearly, this amounts to characterizing the closed translation invariant subspaces of $B$. This is done in 3.1 and 3.2.

Assume that $\mathcal{E}=\left\{E_{\sigma}: \sigma \in \Sigma\right\}$ is defined as in 2.6. For each $\sigma$ let $Y_{\sigma}$ denote a subspace of $H_{\sigma}$ which contains $E_{\sigma}$, and let $\mathcal{Y}$ denote the set $\mathscr{Y}=\left\{Y_{\sigma}: \sigma \in \Sigma\right\}$.

\subsection{Proposition. $B_{0}$ is a homogeneous Banach subspace of $B$.}

Proof. Since ${ }_{x} \hat{b}(\sigma)=U_{\sigma}(x) \hat{b}(\sigma)$ for each $\sigma \in \Sigma$ and $x \in G$, it is easily checked that $B_{\mathscr{y}}$ is translation invariant. So, as $B_{\mathscr{y}}$ inherits the norm of $B$, it is sufficient to prove that it is closed in $B$. Therefore, let $\left(b_{n}\right)_{n \in \mathbf{N}}$ 
denote a sequence in $B_{\mathscr{y}}$ which converges to an element $b$ of $B$. The sequence $\left(\hat{b}_{n}(\sigma)\right)_{n \in \mathrm{N}}$ converges to $\hat{b}(\sigma)$ in $B\left(H_{\sigma}\right)$ for each $\sigma \in \Sigma$. However, for each $n \in \mathbf{N}$ and $\sigma \in \Sigma, Y_{\sigma}$ is a subspace of $\operatorname{ker} \hat{b}_{n}(\sigma)$ and so it must also be a subspace of $\operatorname{ker} \hat{b}(\sigma)$. Therefore $b \in B_{\text {ชy }}$.

3.2 Proposition. Any homogeneous subspace $A$, of $B$, has the form $B_{\circlearrowleft \text {. }}$.

Proof. By 2.6 there exists a set $\mathcal{Y}$ of linear spaces such that $A \cap T(G)$ $=T(G)_{\mathscr{O}}$. In view of $2.4 T(G)_{\mathscr{O}}$ is a dense subspace of $A$, that is, $A$ is the closure in $B$ of $T(G)_{\mathscr{y}}\left(A=\mathrm{cl}_{B} T(G)_{y}\right)$. However, by $3.1, B_{0}$ is a homogeneous Banach subspace of $B$ and so $B_{\mathscr{Y}} \cap T(G)$ is dense in $B_{\mathscr{O}}$. But $B_{\text {ชy }} \cap T(G)=T(G)_{\text {ชy }}$ and so we have $A=B_{\text {ช. }}$.

There are not, in fact, as many 'small' homogeneous Banach spaces (ones which contain only measures) as one might expect.

3.3 Proposition. Assume that each element $b$ of $B$ is a measure. Then $b \in L^{1}(G)$. Moreover, there exists a positive constant $C^{\prime}$ such that $\|b\|_{L^{1}} \leq$ $C^{\prime}\|b\|_{B}$ for all $b \in B$.

Proof. Consider the embedding $L: b \rightarrow b$ of $B$ into $M(G)$. We use the Closed Graph theorem to show that $L$ is continuous and so that there exists a constant $C^{\prime}$ such that $\|b\|_{M} \leq C^{\prime}\|b\|_{B}$ for all $b \in B$.

Let $\left(b_{n}, b_{n}\right)$ denote a sequence taken from the graph of $L$. Assume that there exists $b \in B$ and $\mu \in M(G)$ such that

$$
\lim _{n \rightarrow \infty}\left\|b_{n}-b\right\|_{B}=0 \text { and } \lim _{n \rightarrow \infty}\left\|b_{n}-\mu\right\|_{M}=0 .
$$

Then, for each $\sigma \in \Sigma$

$$
\lim _{n \rightarrow \infty}\left\|\hat{b}_{n}(\sigma)-\hat{b}(\sigma)\right\|_{B\left(H_{\sigma}\right)}=0 \text { and } \lim _{n \rightarrow \infty}\left\|\hat{b}_{n}(\sigma)-\hat{\mu}(\sigma)\right\|_{B\left(H_{\sigma}\right)}=0 .
$$

Consequently $\hat{b}(\sigma)=\hat{\mu}(\sigma)$ for each $\sigma \in \Sigma$ and so $b=\mu$. Thus, the graph of $L$ is closed in $B \times M(G)$, and, by the Closed Graph Theorem, $L$ must be continuous.

To complete the proof we note that the only measures $\mu$ for which the left shift $x \rightarrow_{x} \mu$ is continuous from $G$ to $M(G)$ are those generated by elements of $L^{1}(G)$. For such a $\mu,\|\mu\|_{M}=\|\mu\|_{L^{1}}$.

3.4. COROllaARY TO 2.2 AND 3.3. Every such homogeneous Banach space is a Banach algebra. 
The study of homogeneous Banach algebras is a very rewarding and fruitful one. However we leave this till [13]. See also [8] for the abelian case.

4. Norms. Propositions 2.6, 3.1 and 3.2 demonstrate that there is a close connection between homogeneous Banach spaces and sets of trigonometric polynomials of the form $T(G)_{\mathscr{E}}$. In this section we prove that the homogeneous Banach space $B$ is completely determined by the set $\mathcal{E}$ (where $B \cap T(G)=T(G)_{\mathscr{E}}$ ) and the norm $N$ on $T(G)_{\mathscr{E}}$ (where $N=\|\|_{B}$ restricted to $\left.T(G)_{\mathscr{E}}\right)$. We characterize those norms $N$ which may arise. See also [6].

Given $B$ we already know precisely what $\mathcal{E}$ is (see 2.6 ). We now give some obvious properties of $N$.

4.1. Proposition. (i) $\|b\|_{P} \leq C^{\prime} N(b)$ for all $b \in T(G)_{\mathscr{E}}$.

(ii) Each left-translation operator is a continuous endomorphism of $T(G)_{\mathcal{E}}$ with respect to $N$; moreover each left shift $x \rightarrow_{x} b\left(b \in T(G)_{\mathscr{E}}\right)$ is continuous from $G$ to $T(G)_{\mathcal{E}}$.

We also have:

4.2 Proposition. Let $\left(b_{n}\right)_{n \in \mathbf{N}}$ be a sequence in $T(G)_{\mathfrak{G}}$ which converges to 0 in $P(G)$ and is Cauchy with respect to $N$. Then $\lim _{n \rightarrow \infty} N\left(b_{n}\right)=0$.

Proof. Since the sequence must be Cauchy in $B$ it converges to an element $b$ of $B$. Hence it converges to $b$ in $P(G)$. Thus $b=0$, and we have $\lim _{n \rightarrow \infty} N\left(b_{n}\right)=0$.

Any norm $N$ on $T(G)_{\mathscr{E}}$ which possesses the properties in 4.1 and 4.2 determines a homogeneous Banach space. We must demonstrate how this space is obtained from $N$ and $T(G)_{\mathscr{E}}$. We first introduce some notation.

An element $S \in P(G)$ has property $\mathcal{P}$ if there exists a sequence $\left(b_{n}\right)_{n \in \mathrm{N}}$ of elements of $T(G)_{\tilde{E}}$, Cauchy with respect to $N$, such that $\lim _{n \rightarrow \infty}\left\|S-b_{n}\right\|_{P}=0$. (Obviously $\mathcal{P}$ depends on both $N$ and $\mathcal{E}$, but we will take these to be specified.)

The way in which a homogeneous Banach space is determined by $N$ and $\mathcal{E}$ is suggested by 4.3 .

\subsection{THEOREM.}

$$
B=\{S \in P(G): S \text { has property } \mathcal{P}\} .
$$

Moreover, for each $b \in B$ and every sequence $\left(b_{n}\right)_{n \in \mathrm{N}}$, as in $\mathscr{P},\|b\|_{B}=$ $\lim _{n \rightarrow \infty} N\left(b_{n}\right)$. 
Proof. By $2.6 T(G)_{\varepsilon}$ is dense in $B$. Consequently for each $b \in B$ there exists a sequence $\left(b_{n}\right)_{n \in \mathbf{N}}$ in $T(G)_{\mathscr{E}}$ such that $\lim _{n \rightarrow \infty}\left\|b-b_{n}\right\|_{B}=0$. Obviously $\left(b_{n}\right)_{n \in \mathbf{N}}$ must be Cauchy and must converge to $b$ in $P(G)$. Hence $b$ has property $\mathscr{P}$ and $B$ is included in the set given in (1).

To prove that the reverse inclusion holds let $S$ be an element of $P(G)$ which has property $\mathscr{P}$. Let $\left(b_{n}\right)_{n \in \mathbf{N}}$ denote a sequence from $T(G)_{\mathfrak{E}}$, Cauchy with respect to $N$, and converging to $S$ in $P(G)$. It must be Cauchy in $B$ and so there exists $b \in B$ such that $\lim _{n \rightarrow \infty}\left\|b-b_{n}\right\|_{B}=0$. Since $\left(b_{n}\right)_{n \in \mathbf{N}}$ must also converge to $b$ in $P(G)$, we have $b=S$; that is, $S \in B$.

Before completing the proof of 4.3 we require a lemma.

4.4 Lemma. Let $S$ be an element of $P(G)$ with property $\mathcal{P}$. If $\left(b_{n}\right)_{n \in \mathrm{N}}$ and $\left(c_{n}\right)_{n \in \mathrm{N}}$ denote two sequences as in $\mathscr{P}$ then

$$
\lim _{n \rightarrow \infty} N\left(b_{n}\right)=\lim _{n \rightarrow \infty} N\left(c_{n}\right) .
$$

Proof. We define the new sequence $\left(d_{n}\right)_{n \in \mathrm{N}}$ by $d_{n}=b_{n}-c_{n}$. Clearly each $d_{n} \in T(G)_{\tilde{E}}$ and the sequence converges to 0 in $P(G)$. It is Cauchy with respect to $N$ and so converges to 0 in $T(G)_{\mathscr{E}}$. Therefore

$$
\lim _{n \rightarrow \infty} N\left(b_{n}\right)=\lim _{n \rightarrow \infty} N\left(c_{n}\right) .
$$

Proof of 4.3 continued. Let $b \in B$ and $\left(b_{n}\right)_{n \in \mathbf{N}}$ denote a sequence taken from $T(G)_{\mathscr{E}}$, Cauchy with respect to $N$, and converging to $b$ in $P(G)$. In view of 4.4 it is sufficient to prove that $\|b\|_{B}=\lim _{n \rightarrow \infty} N\left(b_{n}\right)$. The sequence $\left(b_{n}\right)_{n \in \mathrm{N}}$ must converge to $b$ in $B$. Moreover, for each $n$,

$$
\left|\|b\|_{B}-\left\|b_{n}\right\|_{B}\right|=\left|\|b\|_{B}-N\left(b_{n}\right)\right| \leq\left\|b-b_{n}\right\|_{B}
$$

and so the result follows.

We now complete the classification of homogeneous Banach spaces.

Let $\mathcal{E}$ denote a set of linear spaces, $\mathcal{E}=\left\{E_{\sigma} \subseteq H_{\sigma}, \sigma \in \Sigma\right\}$, and let $N$ denote a norm on $T(G)_{\mathfrak{E}}, N$ having the properties listed in 4.1 and 4.2. We determine a homogeneous Banach space $B$, with norm \|\|$_{B}$, such that $B \cap T(G)=T(G)_{\mathscr{E}}$ and such that \|\|$_{B}$ and $N$ coincide on $T(G)_{\mathscr{E}}$. In this way we establish a correspondence between homogeneous Banach spaces and the pairs $(N, \mathcal{E})$.

$\operatorname{Regard}(N, \mathcal{E})$ as fixed. 
Put $B=\{S \in P(G): S$ has property $\mathscr{P}\}$, and for each $S \in B$ put $\|S\|_{B}=\lim _{n \rightarrow \infty} N\left(b_{n}\right)$ (by 4.4\|\|$_{B}$ is single-valued). Clearly $T(G)_{\mathfrak{E}}$ is a dense subset of $B$.

We prove that $\left(B,\|\|_{B}\right)$ is a suitable homogeneous Banach space. First we show that it is a Banach space, then that it is homogeneous, and finally that it is suitable.

\subsection{Proposition. $\left(B,\|\|_{B}\right)$ is a Banach space.}

Proof. Clearly $B$ is a linear space and \|\|$_{B}$ is a seminorm. Therefore $B$ is a normed space if the only $S \in B$ with zero norm is $S=0$. To see that this is so, let $\left(b_{n}\right)$ be a sequence in $T(G)_{\mathscr{E}}$, Cauchy with respect to $N$, and converging to $S$ in $P(G)$. Then as $\|S\|_{B}=0$, we have $\lim _{n \rightarrow \infty} N\left(b_{n}\right)=0$, and so $\lim _{n \rightarrow \infty}\left\|b_{n}\right\|_{P}=0$. Therefore $\left(b_{n}\right)_{n \in \mathrm{N}}$ converges to 0 in $P(G)$, and so $S=0$.

Finally, we show that $B$ is complete. Let $\left(S_{n}\right)_{n \in \mathbf{N}}$ denote a Cauchy sequence in $B$. For each $n$ there exists $b_{n} \in T(G)_{\mathfrak{G}}$ such that

$$
\left\|S_{n}-b_{n}\right\|_{B}<1 / n \text {. }
$$

Given $\varepsilon>0$ there exists $N \in \mathbf{N}$ such that for $m, n>N$,

$$
\left\|S_{n}-S_{m}\right\|_{B}<\varepsilon
$$

Because

$$
N\left(b_{n}-b_{m}\right) \leq\left\|b_{n}-S_{n}\right\|_{B}+\left\|S_{n}-S_{m}\right\|_{B}+\left\|S_{m}-b_{m}\right\|_{B}
$$

the sequence $\left(b_{n}\right)_{n \in \mathrm{N}}$ is Cauchy with respect to $N$. Now there exists $S \in P(G)$ such that $\left(S_{n}\right)_{n \in \mathbf{N}}$ converges to $S$ in $P(G)$. Obviously $\left(b_{n}\right)_{n \in \mathbf{N}}$ also converges to $S$ in $P(G)$ and so $S \in B$. We need only prove that $\lim _{n \rightarrow \infty}\left\|S-S_{n}\right\|_{B}=0$. For a fixed $m$ the sequence $\left(b_{n}-b_{m}\right)_{n \in \mathbf{N}}$ is Cauchy with respect to $N$ and converges to $S-b_{m}$ in $P(G)$. Hence $S-b_{m} \in B$, and

$$
\left\|S-b_{m}\right\|_{B}=\lim _{n \rightarrow \infty} N\left(b_{n}-b_{m}\right)=\varepsilon_{m} \quad \text { say. }
$$

As $\left(b_{n}\right)_{n \in \mathbf{N}}$ is Cauchy with respect to $N$ we have $\lim _{m \rightarrow \infty} \varepsilon_{m}=0$. Hence $\lim _{n \rightarrow \infty}\left\|S-S_{n}\right\|_{B}=0$.

\subsection{Proposition. $B$ is homogeneous.}

Proof. The set $T(G)_{\mathcal{E}}$ is left-translation invariant. Moreover each left translation operator is an isometry of $P(G)$ and so $B$ is translation 
invariant. Each translation operator is a continuous endomorphism of $T(G)_{\mathscr{E}}$ and so also of $B$; similarly the map $x \rightarrow{ }_{x} S$ is continuous from $G$ to $T(G)_{\mathfrak{E}}$ (and so to $B$ ) for each $S \in T(G)_{\mathfrak{E}}$. Consequently the Baire Category theorem may be used to show that $x \rightarrow{ }_{x} S$ is continuous from $G$ to $B$ for all $S \in B$.

4.7. Proposition. $B \cap T(G)=T(G)_{\tilde{G}}$ and \|\|$_{B}$ and $N$ coincide on $T(G)_{\mathscr{E}}$.

Proof. Clearly $N$ and \|\|$_{B}$ coincide on $T(G)_{\mathfrak{E}}$ because for each $b \in T(G)_{\mathfrak{E}}$ the constant sequence $(b)_{n \in \mathrm{N}}$ is Cauchy with respect to $N$ and converges to $b$ in $P(G)$.

By $2.6 B \cap T(G)=T(G)_{\mathscr{\partial}}$ for some set $\mathscr{Y}=\left\{Y_{\sigma} \subseteq H_{\sigma}, \sigma \in \Sigma\right\}$. As $T(G)_{\mathcal{E}} \subseteq B \cap T(G)$ we consider the case: there exists $\sigma \in \Sigma$ such that $Y_{\sigma}$ is a proper subspace of $E_{\sigma}$. There exists $b \in B \cap T(G)$ such that $\hat{b}(\sigma)\left(E_{\sigma}\right)$ $\neq\{0\}$. Choose $\xi \in E_{\sigma}$ such that $\hat{b}(\sigma) \xi \neq 0$. Now there exists a sequence $\left(b_{n}\right)_{n \in \mathbf{N}}$ in $T(G)_{\mathscr{E}}$, Cauchy with respect to $N$, such that $\left(b_{n}\right)_{n \in \mathbf{N}}$ converges to $b$ in $P(G)$. However,

$$
\left\|b-b_{n}\right\|_{P} \geq\left\|\hat{b}(\sigma)-\hat{b}_{n}(\sigma)\right\|_{B\left(H_{\mathrm{o}}\right)} \geq \sup _{\zeta \in E_{\sigma}}\|\hat{b}(\sigma) \zeta\|_{H_{\sigma}}>0,
$$

a contradiction. Hence $\mathcal{E}=\mathcal{Y}$.

5. Examples. As we have already stated each of the Banach spaces $A(G), C(G), L^{p}(G), 1 \leq p<\infty$, and $P F(G)$, when equipped with its usual norms, is homogeneous. We now consider some more examples.

For each $p, 1 \leq p<\infty$, let $U^{p}(G)=\left\{f \in L^{1}(G): \hat{f} \in E_{p}(\Sigma)\right\}$ and let $\|f\|_{U^{p}}=\|f\|_{L^{1}}+\|\hat{f}\|_{p}$ for each $f \in U^{p}(G)$. It is shown in 38.28 of [4] that $U^{P}(G)$ is a Banach algebra under convolution. It contains $T(G)$ as a dense subspace and so must be left translation invariant (because $T(G)$ is).

\subsection{Proposition. $U^{p}(G)$ is homogeneous.}

Proof. We need only show that each shift is bounded and that the map $x \rightarrow_{x} f$, from $G$ to $U^{p}(G)$, is continuous at the identity $e$.

Let $x \in G$. Then

$$
\left\|_{x} f\right\|_{U^{p}}=\left\|_{x} f\right\|_{L^{1}}+\left\|_{x} \hat{f}\right\|_{p} \leq\|f\|_{L^{1}}+\left\|U_{x} \hat{f}\right\|_{p}
$$

where. $U_{x}(\sigma)=U_{\sigma}(x)$ for each $\sigma \in \Sigma$. Now $U_{x} \in E_{\infty}(\Sigma)$; in fact $\left\|U_{x}\right\|_{\infty}=$ 1 , and so

$$
\left\|_{x} f\right\|_{U^{p}} \leq\|f\|_{L^{1}}+\|\hat{f}\|_{p}=\|f\|_{U^{p}} .
$$

Therefore each shift is bounded. 
Now it is clear that the map $x \rightarrow_{x} f$ is continuous at $e$ if the map $x \rightarrow{ }_{x} \hat{f}$, from $G$ to $E_{p}(\Sigma)$, is continuous at $e$. But we have

$$
\left\|{ }_{x} \hat{f}-\hat{f}\right\|_{p}^{p}=\sum_{\sigma \in \Sigma} d_{\sigma}\left\|U_{\sigma}(x) \hat{f}(\sigma)-\hat{f}(\sigma)\right\|_{\phi_{p}}^{p} .
$$

For each $\varepsilon>0$ there exists a finite subset $F$ of $\Sigma$ such that

$$
\sum_{\sigma \in \Sigma \backslash F} d_{\sigma}\|\hat{f}(\sigma)\|_{\phi_{p}}^{p}<\varepsilon^{p} .
$$

Since $U_{\sigma}$ is a continuous representation (1) and (2) together prove the result.

Again let $F$ denote a subset of $\Sigma$. It is easy to see that $E(\Sigma)_{F}$ (equal to those elements of $E(\Sigma)$ supported by $F$ ) and $E(F)$ (equal to $\prod_{\sigma \in F} B\left(H_{\sigma}\right)$ ) are isomorphic. Hence we identify the two linear spaces.

Let $A=\left(A_{\sigma}\right)_{\sigma \in \Sigma}$ be an element of $E(F)$. Assume that each $A_{\sigma}$ is invertible and that $A^{-1}=\left(A_{\sigma}^{-1}\right)_{\sigma \in \Sigma}$ is in $E_{\infty}(F)$. For any $p \in\{0\} \cup$ $[1, \infty]$ let

$$
P(G)^{A, p}=\left\{S \in P(G)_{F}: \hat{S} A \in E_{p}(F)\right\} .
$$

For each $S \in P(G)^{A, p}$ let $\|S\|_{A, p}=\|\hat{S} A\|_{p}$. Then $\left(P(G)^{A, p},\|\|_{A, p}\right)$ is a normed space. It is a generalization of the Segal algebra $S(\alpha)$ studied by M. Riemersma in [8] and of the Segal algebra $A_{p}(G)$ studied by Larsen, Liu and Wang in [5], in both cases for abelian groups.

5.2. Proposition. If $p \neq \infty$ then $P(G)^{A, p}$ is homogeneous.

Proof. We first prove that the map $S \rightarrow S$, of $P(G)^{A, p}$ to $P(G)$, is continuous. For any $S \in P(G)^{A, p}$

$$
\|S\|_{A, p}=\|\hat{S} A\|_{p} \geq\|\hat{S} A\|_{\infty}=\sup _{\sigma \in F}\left\|\hat{S}(\sigma) A_{\sigma}\right\|_{B\left(H_{\sigma}\right)}
$$

But

$$
\left\|\hat{S}(\sigma) A_{\sigma}\right\|_{B\left(H_{\sigma}\right)} \geq\|\hat{S}(\sigma)\|_{B\left(H_{\sigma}\right)}\left\|A_{\sigma}^{-1}\right\|_{B\left(H_{\sigma}\right)}^{-1} \geq\|\hat{S}(\sigma)\|_{B\left(H_{\sigma}\right)}\left[\sup _{\sigma \in F}\left\|A_{\sigma}^{-1}\right\|_{B\left(H_{\sigma}\right)}\right]^{-1} .
$$

So it is continuous.

To prove that $P(G)^{A, p}$ is a Banach space it is sufficient to prove that it is complete. If $\left(S_{n}\right)_{n \in \mathrm{N}}$ is a Cauchy sequence in $P(G)^{A, p}$ then it is also Cauchy in $P(G)$. Therefore it converges in $P(G)$ to an element $S$. Let 
$\left(S_{n_{j}}\right)_{n \in \mathbf{N}}$ be a subsequence chosen so that for each $j$

$$
\left\|S_{n_{j+1}}-S_{n_{j}}\right\|_{A, p}<2^{-j}
$$

For each $N \in \mathbf{N}$

$$
S_{n_{N}}=S_{n_{1}}+\sum_{j=1}^{N-1}\left(S_{n_{J+1}}-S_{n_{J}}\right)
$$

and so

$$
\left\|S_{n_{N}}\right\|_{A, p} \leq\left\|S_{n_{1}}\right\|_{A, p}+\sum_{j=1}^{N-1}\left\|S_{n_{j+1}}-S_{n_{j}}\right\|_{A, p} \leq\left\|S_{n_{1}}\right\|_{A, p}+\sum_{j=1}^{N-1} 2^{-\jmath} .
$$

Therefore $S \in P(G)^{A, P}$.

It is easily seen that $P(G)^{A, p}$ is left translation invariant and that each shift is continuous. Moreover the argument used in 5.1 can show that the map $x \rightarrow{ }_{x} S$, from $G$ to $P(G)^{A, p}$, is continuous at $e$. Therefore $P(G)^{A, p}$ is homogeneous.

Clearly $\left\{\hat{S}: S \in P(G)^{A, p}\right\}$ is a subset of $E_{p}(F)$. If $A$ is an element of $E_{\infty}(F)$ then these two sets are equal. Further it is interesting to note that if $A^{-1}$ is an element of $E^{q}(F)$ then $\left\{\hat{S}: S \in P(G)^{A, p}\right\}$ is a subset of $E^{r}(F)$ (where $1 / r=1 / p+1 / q$ if $p$ and $q$ are non-zero, $1 / r=1 / p$ if $q=0$ and $1 / r=1 / q$ if $p=0)$.

\section{REFERENCES}

[1] J. T. Burnham, Closed ideals in subalgebras of Banach algebras I, Proc. Amer. Math. Soc., 32 (1972), 551-555.

[2] Segal Algebras and Dense Ideals in Banach Algebras, Springer-Verlag, Lecture Notes in Math., 399 (1974), 33-58.

[3] R. E. Edwards, Functional Analysis, Theory and Applications, Holt, Rinehart and Winston Inc., New York, 1965.

[4] E. Hewitt and K. A. Ross, Abstract Harmonic Analysis, 2 vols. Springer-Verlag, Berlin, 1963 and 1970.

[5] R. Larsen, T. S. Liu and J. K. Wang, On the functions with Fourier transforms in $L^{p}$, Michigan Math. J., 11 (1964), 369-378.

[6] K. de Leeuw, Homogeneous algebras on compact abelian groups, Trans. Amer. Math. Soc., 87 (1958), 372-386.

[7] H. Reiter, $L^{1}$-algebras and Segal algebras, Lecture Notes in Math., 231, 1971.

[8] M. Riemersma, On some properties of normed ideals in $L^{1}(G)$, Indag. Math., 37 (1975), 265-272.

[9] G. E. Silov, Homogeneous rings of functions, Amer. Math. Soc. Trans., ser. 1, 8 (1954), 393-455.

[10] H. C. Wang, Homogeneous Banach algebras, Lecture Notes in Pure and Appl. Math., Dekker, 1977. 
[11] J. A. Ward, Banach spaces of pseudomeasures on compact groups with emphasis on homogeneous spaces, Ph.D. thesis, Australia National University, 1979.

[12] Ideal structure of operator and measure algebras, Monat. Math., 95 (1983), 159-172.

[13] _ Ideal structure of homogeneous algebras, Monat. Math., 96 (1983), 317-324.

Received September 24, 1982.

MURDOCH UNIVERSITY

MURDOCH, WeSTERN AUSTRALIA 6150

AUSTRALIA 



\section{PACIFIC JOURNAL OF MATHEMATICS EDITORS}

\author{
DONALD BABBITT (Managing Editor) \\ University of California \\ Los Angeles, CA 90024 \\ J. DugundJI \\ University of Southern California \\ Los Angeles, CA 90089-1113 \\ R. FINN \\ Stanford University \\ Stanford, CA 94305 \\ HERMANN FLASChKa \\ University of Arizona \\ Tucson, AZ 85721
}

C. C. MOORE

University of California

Berkeley, CA 94720

Arthur Ogus

University of California

Berkeley, CA 94720

Hugo RossI

University of Utah

Salt Lake City, UT 84112

H. SAMELSON

Stanford University

Stanford, CA 94305

ASSOCIATE EDITORS
R. ARENS
E. F. BECKENBACH
B. H. NeUmanN
F. WOLF
K. YosHIDA (1906-1982)

\section{SUPPORTING INSTITUTIONS}
UNIVERSITY OF ARIZONA
UNIVERSITY OF BRITISH COLUMBIA
CALIFORNIA INSTITUTE OF TECHNOLOGY
UNIVERSITY OF CALIFORNIA
MONTANA STATE UNIVERSITY
UNIVERSITY OF NEVADA, RENO
NEW MEXICO STATE UNIVERSITY
OREGON STATE UNIVERSITY

\author{
UNIVERSITY OF OREGON \\ UNIVERSITY OF SOUTHERN CALIFORNIA \\ STANFORD UNIVERSITY \\ UNIVERSITY OF HAWAII \\ UNIVERSITY OF TOKYO \\ UNIVERSITY OF UTAH \\ WASHINGTON STATE UNIVERSITY \\ UNIVERSITY OF WASHINGTON
}

The Supporting Institutions listed above contribute to the cost of publication of this Journal, but they are not owners or publishers and have no responsibility for its content or policies.

Mathematical papers intended for publication in the Pacific Journal of Mathematics should be in typed form or offset-reproduced (not dittoed), double spaced with large margins. Please do not use built up fractions in the text of the manuscript. However, you may use them in the displayed equations. Underline Greek letters in red, German in green, and script in blue. The first paragraph must be capable of being used separately as a synopsis of the entire paper. In particular it should contain no bibliographic references. Please propose a heading for the odd numbered pages of less than 35 characters. Manuscripts, in triplicate, may be sent to any one of the editors. Please classify according to the scheme of Math. Reviews, Index to Vol. 39. Supply name and address of author to whom proofs should be sent. All other communications should be addressed to the managing editor, or Elaine Barth, University of California, Los Angeles, California 90024.

There are page-charges associated with articles appearing in the Pacific Journal of Mathematics. These charges are expected to be paid by the author's University, Government Agency or Company. If the author or authors do not have access to such Institutional support these charges are waived. Single authors will receive 50 free reprints; joint authors will receive a total of 100 free reprints. Additional copies may be obtained at cost in multiples of 50 .

The Pacific Journal of Mathematics is issued monthly as of January 1966. Regular subscription rate: $\$ 190.00$ a year (5 Vols., 10 issues). Special rate: $\$ 66.00$ a year to individual members of supporting institutions.

Subscriptions, orders for numbers issued in the last three calendar years, and changes of address should be sent to Pacific Journal of Mathematics, P.O. Box 969, Carmel Valley, CA 93924, U.S.A. Old back numbers obtainable from Kraus Periodicals Co., Route 100, Millwood, NY 10546.

The Pacific Journal of Mathematics at P.O. Box 969, Carmel Valley, CA 93924 (ISSN 0030-8730) publishes 5 volumes per year. Application to mail at Second-class postage rates is pending at Carmel Valley, California, and additional mailing offices. Postmaster: Send address changes to Pacific Journal of Mathematics, P.O. Box 969, Carmel Valley, CA 93924.

PUBLISHED BY PACIFIC JOURNAL OF MATHEMATICS, A NON-PROFIT CORPORATION

Copyright $\odot 1984$ by Pacific Journal of Mathematics 


\section{Pacific Journal of Mathematics}

Vol. 114, No. $2 \quad$ June, 1984

William Allen Adkins, A Harnack estimate for real normal surface

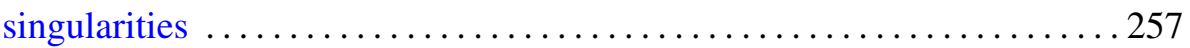

George E. Andrews, Multiple series Rogers-Ramanujan type identities . . . . 267

Didier Arnal, $*$ products and representations of nilpotent groups . . . . . . 285

David Cox and Walter Raymond Parry, Representations associated with

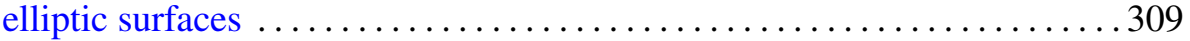

Joanne Marie Dombrowski, Tridiagonal matrix representations of cyclic

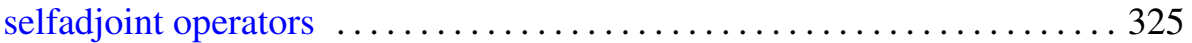

Ronald Dotzel, An Artin relation $(\bmod 2)$ for finite group actions on

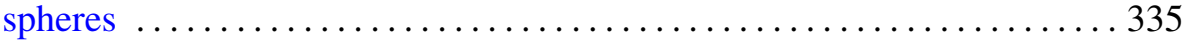

Leo Egghe, Convergence of adapted sequences of Pettis-integrable

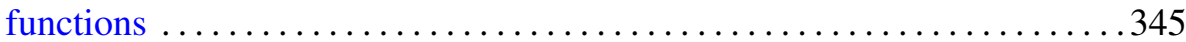

Rebecca A. Herb, Characters of induced representations and weighted

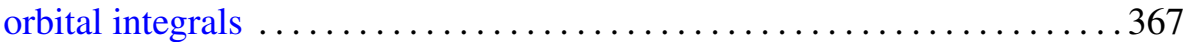

Steven M. Kahn, Cobordism obstructions to fibering manifolds over

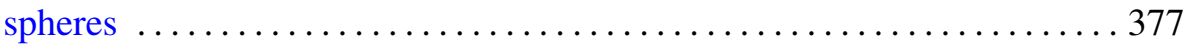

Robert D. Little, Projective space as a branched covering of the sphere with orientable branch set

Claude Schochet, Topological methods for $C^{*}$-algebras. III. Axiomatic

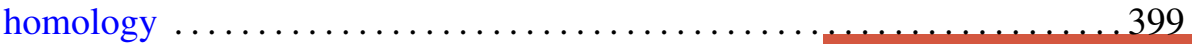

Claude Schochet, Topological methods for $C^{*}$-algebras. IV. $\bmod p$ homology

James M. Stormes, On the $K O$-orientability of complex projective varieties

Josephine Anne Ward, Characterization of homogeneous spaces and their norms 